\title{
Non-Newtonian Effects of Rabinowitsch Fluid on the Performance of Inclined Stepped Composite Bearings
}

\author{
Naduvinamani Neminath Bhujappa ${ }^{1)^{*}}$ and Rajashekar Mareppa ${ }^{2)}$ \\ ${ }^{1)}$ Department of Mathematics, Gulbarga University \\ Gulbarga-585106, India \\ ${ }^{2)}$ Department of Mathematics, Govt. PU. College for Girls \\ Raichur-584101, India \\ *Corresponding author: naduvinamaninb@yahoo.co.in
}

( Manuscript received 13 December 2012; accepted 15 May 2013; published 15 June 2013 )

\begin{abstract}
The theoretical analysis of rheological effects of Rabinowitsch fluid on the steady and dynamic characteristics of inclined stepped composite bearings is investigated. The Rabinowitsch fluid model is considered to account the pseudoplastic and dilatant nature of the lubricant due to the presence of additives. The perturbation technique is used to derive the modified Reynolds equation separately for both steady state and perturbed characteristics of the bearing. The closed form expressions for the bearing characteristics are obtained. By using these expressions, the performance characteristics of four different types of bearings such as stepped, plane inclined slider, composite tapered land and composite tapered concave bearings are determined. It is found that, the non-Newtonian behaviour of the Rabinowitsch fluid have a significant effect on bearing characteristics. Further, it is found that the existence of a critical value for profile parameter at which the dynamic stiffness coefficient attains maximum.
\end{abstract}

Keywords: inclined stepped composite bearings, steady state characteristics, dynamic characteristics, non-Newtonian lubricants, Rabinowitsch fluid model

\section{Introduction}

Nowadays, the use of Newtonian fluids blended with various additives increases due to their effective improvement in the bearing characteristics as compared to the Newtonian lubricants. Due to the addition of additives to the Newtonian lubricant, the non-linear relationship exists between the shear stress and shear strain rate. There are several fluid models to study the non-Newtonian properties of the lubricants such as power law, couple stress and micropolar fluid model. Maiti [1] analyzed composite and step slider bearings with micropolar fluid as lubricant. The effect of additives in the lubricant of a composite bearing with an inclined stepped surface was studied by Prawal Sinha [2]. The effects of increased load-carrying capacity and decrease in the coefficient of friction were found in their study. Naduvinamani et al. analyzed inclined stepped composite bearings with micropolar fluid [3], slider bearings with micropolar fluid [4] and slider bearings with couple stress fluid [5]. Bhat and Daheri [6] investigated porous composite slider bearings lubricated with magnetic fluid. It is reported that, the magnetic fluid increases the load capacity and decreases the coefficient of friction. Shukla and Isa [7] investigated the characteristics of non-Newtonian power law lubricants in step bearings and hydrostatic step seals. Apart from these, Rabinowitsch fluid model is also one such fluid model which can be applied to analyze the non-linear behavior of non-Newtonian lubricants.

In the Rabinowitsch fluid model, the non-linear relationship between shear stress and shear strain rate can be described for one-dimensional fluid flow as follows

$$
\tau_{x y}+\kappa \tau_{x y}^{3}=\mu \frac{\partial u}{\partial y}
$$

where $\mu$ denotes the zero shear rate viscosity and $\kappa$ denotes the non-linear factor which describes the non-Newtonian effects of the lubricant. By using this fluid model, one can analyze the Newtonian fluids $(\kappa=0)$, dilatant fluids $(\kappa<0)$ and pseudoplastic fluids $(\kappa>0)$. The theoretical analysis of this fluid model agrees well with the experimental results conducted by Wada and Hayashi [8]. Determination of the load capacity of finite 
width journal bearing by finite element method in the case of a non-Newtonian lubricant was analyzed by Bourgin and Gay [9]. Hashimoto and Wada [10] studied the effects of fluid inertia forces in parallel circular squeeze film bearing lubricated with pseudoplastic fluids. Recently, several researchers have investigated the non-Newtonian effects of Rabinowitsch lubricants on various types of bearings. Lin et al. studied the non-Newtonian effects of Rabinowitsch fluid model on the slider bearings [11], parallel annular disks [12] and parallel rectangular squeeze-film plates [13] respectively. Variational principal for non-Newtonian lubrication: Rabinowitsch fluid model analyzed by He [14]. Singh et al. investigated the effects of Rabinowitsch fluid model on the hydrostatic thrust bearing [15], annular ring hydrostatic thrust bearing [16] and a squeeze film characteristics between a long cylinder and a flat plate [17].

In this paper, an attempt has been made to analyze the steady-state and dynamic characteristics of inclined stepped composite bearings lubricated with Rabinowitsch fluid which has not been studied so far.

\section{Mathematical Formulation and solution of the problem}

The physical configuration for different types of bearings is shown in Figure 1. The geometry consists of two surfaces separated by a non-Newtonian lubricant, namely, Rabinowitsch fluid. The lower surface is moving with a velocity $U$ in $x$-direction and the lubricant in the film region is taken along $y$-axis.

The fluid film thickness is composed of two parts

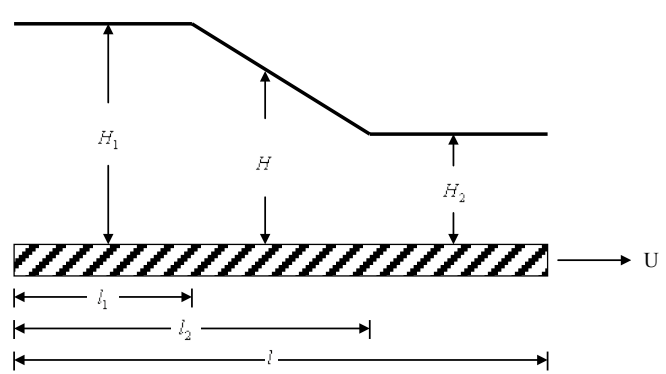

Fig. 1 (a) inclined stepped composite bearing

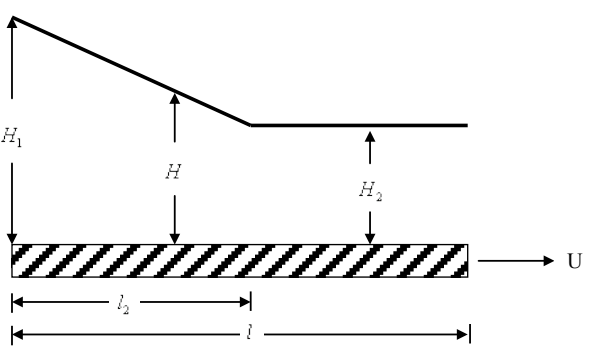

Fig. 1 (c) composite tapered land bearing

$$
\begin{aligned}
h(x) & =h_{m}+h_{s} \\
& = \begin{cases}h_{m}+d & \text { for } \quad 0 \leq x \leq l_{1} \\
h_{m}+d\left(\frac{l_{2}-x}{l_{2}-l_{1}}\right) & \text { for } \quad l_{1} \leq x \leq l_{2} \\
h_{m} & \text { for } \quad l_{2} \leq x \leq 1\end{cases}
\end{aligned}
$$

where $h_{m}$ is minimum film thickness and $h_{s}$ is slider profile function.

The basic equations governing the flow of an incompressible non-Newtonian Rabinowitsch fluid under the assumptions of hydrodynamic lubrication for thin film is given by

$$
\begin{aligned}
& \frac{\partial u}{\partial x}+\frac{\partial v}{\partial y}=0 \\
& \frac{\partial p}{\partial x}=\frac{\partial \tau_{x y}}{\partial y} \\
& \frac{\partial p}{\partial y}=0
\end{aligned}
$$

The relevant boundary conditions for velocity components are

$$
\begin{aligned}
& u=U, \quad v=0, \quad \text { at } \quad y=0 \\
& u=0, \quad v=V, \quad \text { at } \quad y=h
\end{aligned}
$$

Integrating equation (4) with respect to $y$ subject to the boundary conditions (6a) and (6b) and using constitutive equation (1), the expression for velocity component is obtained in the form

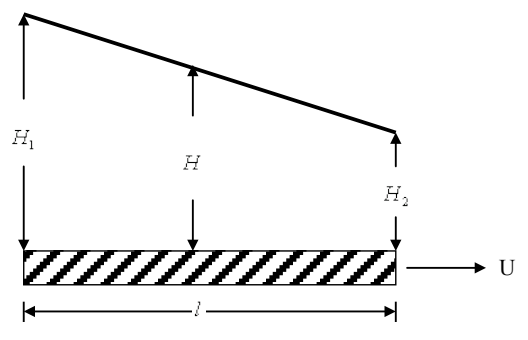

Fig. 1 (b) plane slider bearing

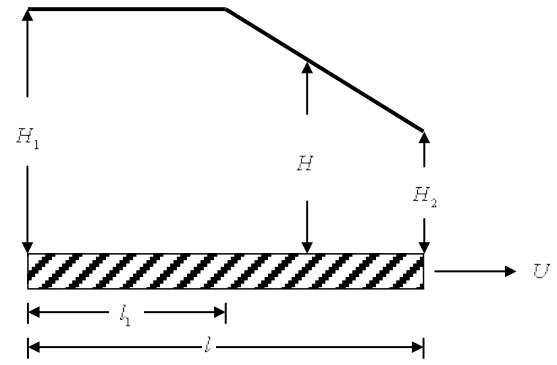

Fig. 1 (d) composite tapered concave bearing 


$$
\begin{aligned}
u= & \frac{1}{\mu}\left[\frac{1}{2} y(y-h)+\kappa^{*} g^{3} F_{1}\right] \\
& +U\left[1-\frac{y+\kappa^{*} g^{2} F_{2}}{h\left(1+0.25 \kappa^{*} g^{2} h^{2}\right)}\right]
\end{aligned}
$$

where

$$
\begin{aligned}
& F_{1}=\frac{1}{4} y^{4}-\frac{1}{2} h y^{3}+\frac{3}{8} h^{2} y^{2}-\frac{1}{8} h^{3} y, \\
& F_{2}=y^{3}-\frac{3}{2} h y^{2}+\frac{3}{4} h^{2} y \text { and } g=\frac{\partial p}{\partial x} .
\end{aligned}
$$

Using equation (7) in continuity equation (3) and integrating with respect to $y$ under the relevant boundary conditions (6a) and (6b) for $y$, the Reynolds type equation for non-Newtonian Rabinowitsch fluid is obtained in the form

$$
\frac{\partial}{\partial x}\left[h^{3} \frac{\partial p}{\partial x}+\frac{3}{20} \kappa^{*} h^{5}\left(\frac{\partial p}{\partial x}\right)^{3}\right]=6 \mu U \frac{\partial h}{\partial x}+12 \mu \frac{\partial h}{\partial t}
$$

Using the non-dimensional parameters

$$
\begin{aligned}
& h^{*}=\frac{h}{h_{m_{0}}} ; \quad x^{*}=\frac{x}{l} ; \quad h_{s}^{*}=\frac{h_{s}}{h_{m_{0}}} ; \\
& h_{m}^{*}=\frac{h_{m}}{h_{m_{0}}} ; \quad \tau=\omega t ; \quad p^{*}=\frac{p h_{m_{0}}^{2}}{\mu U l} ; \\
& \delta=\frac{d}{h_{m_{0}}} ; \quad \sigma=\frac{\omega l}{U} ; \quad \alpha=\frac{\kappa^{*} \mu^{2} U^{2}}{h_{m_{0}}^{2}}
\end{aligned}
$$

Into equations (2) and (8), the dimensionless film thickness for inclined stepped composite bearing is obtained as

$$
h(x)=h_{m}^{*}(\tau)+h_{s}^{*}\left(x^{*}\right)
$$

where $\delta$ is the dimensionless wedge parameter of the slider profile and the non-dimensional form of Reynolds type equation for Rabinowitsch fluid is obtained in the form

$$
\frac{\mathrm{d}}{\mathrm{d} x^{*}}\left[h^{*^{3}} \frac{\mathrm{d} p^{*}}{\mathrm{~d} x^{*}}+\frac{3 \alpha}{20} h^{*^{5}}\left(\frac{\mathrm{d} p^{*}}{\mathrm{~d} x^{*}}\right)^{3}\right]=6 \frac{\mathrm{d} h_{s}^{*}}{\mathrm{~d} x^{*}}+12 \sigma \frac{\mathrm{d} h_{m}^{*}}{\mathrm{~d} \tau}
$$

where $\alpha$ denotes the dimensionless non-linear factor responsible for non-Newtonian effects and $\sigma$ denotes the dimensionless squeeze number.

Equation (10) is a non-linear equation in $p^{*}$, hence it is not easy to find its solution in closed form using analytical methods. Hence, the classical perturbation method is used to find its solution. The dimensionless minimum film thickness and film pressure can be perturbed as

$$
\begin{aligned}
& h_{m}^{*}=1+\varepsilon e^{i \tau} \\
& p^{*}=p_{0}^{*}+\varepsilon p_{1}^{*} e^{i \tau}
\end{aligned}
$$

Substituting into the dimensionless Reynolds-type equation (10) and neglecting the higher order terms of $\varepsilon$, the two Reynolds-type equations corresponding to both steady-state and dynamic stiffness characteristics respectively are obtained in the form

$$
\frac{\mathrm{d}}{\mathrm{d} x^{*}}\left[H^{* 3} \frac{\mathrm{d} p_{0}^{*}}{\mathrm{~d} x^{*}}+\frac{3 \alpha}{20} H^{* 5}\left(\frac{\mathrm{d} p_{0}^{*}}{\mathrm{~d} x^{*}}\right)^{3}\right]=6 \frac{\mathrm{d} H^{*}}{\mathrm{~d} x^{*}}
$$

(steady)

and

$$
\frac{\mathrm{d}}{\mathrm{d} x^{*}}\left[H^{*^{3}}\left\{1+\frac{9 \alpha}{20} H^{* 2}\left(\frac{\mathrm{d} p_{0}^{*}}{\mathrm{~d} x^{*}}\right)^{2}\right\} \frac{\mathrm{d} p_{1}^{*}}{\mathrm{~d} x^{*}}\right]=12 \sigma i
$$

(perturbed)

where

$$
H^{*}=\left\{\begin{array}{lll}
H_{1}^{*}=1+\delta & \text { for } & 0 \leq x^{*} \leq L_{1}^{*} \\
H^{*}=1+\delta\left(\frac{L_{2}^{*}-x^{*}}{L_{2}^{*}-L_{1}^{*}}\right) & \text { for } & L_{1}^{*} \leq x^{*} \leq L_{2}^{*} \\
H_{2}^{*}=1 & \text { for } & L_{2}^{*} \leq x^{*} \leq 1
\end{array}\right.
$$

\subsection{Steady-state characteristics}

The steady-state film pressure can be obtained by solving the steady Reynolds-type equation (13) with the boundary conditions

$$
p_{0}^{*}=0 \quad \text { at } \quad x^{*}=0,1
$$

and the pressure is continuous at $x^{*}=L_{1}{ }^{*}$ and $x^{*}=L_{2}{ }^{*}$.

Observing equation (13), the value of $p_{0}{ }^{*}$ can be obtained numerically, and applied to calculate the perturbed pressure. But it is difficult to solve the non-linear equation (13) analytically for the steady pressure. In order to obtain an analytical approximate expression, a classical perturbation technique is used and the steady pressure $p_{0}{ }^{*}$ is expressed as (for $-0.1<\alpha<0.1)$

$$
p_{0}^{*}=p_{00}^{*}+\alpha p_{01}^{*}
$$

Substitution of equation (17) into equation (13) results in the following two equations governing $p_{00}{ }^{*}$ and $p_{01}{ }^{*}$ :

$$
\begin{aligned}
& \frac{\mathrm{d}}{\mathrm{d} x^{*}}\left[H^{*^{3}} \frac{\mathrm{d} p_{00}^{*}}{\mathrm{~d} x^{*}}\right]=6 \frac{\mathrm{d} H^{*}}{\mathrm{~d} x^{*}} \\
& \frac{\mathrm{d}}{\mathrm{d} x^{*}}\left[\frac{3}{20} H^{*^{5}}\left(\frac{\mathrm{d} p_{00}^{*}}{\mathrm{~d} x^{*}}\right)^{3}+H^{*^{3}} \frac{\mathrm{d} p_{01}^{*}}{\mathrm{~d} x^{*}}\right]=0
\end{aligned}
$$

Once integration of equation (18) with respect to $x^{*}$ gives

$$
\frac{\mathrm{d} p_{00}^{*}}{\mathrm{~d} x^{*}}=6\left(\frac{H^{*}-H_{0}^{*}}{H^{* 3}}\right)
$$

where $H_{0}{ }^{*}$ is the film thickness at which $\frac{\mathrm{d} p_{00}^{*}}{\mathrm{~d} x^{*}}=0$. Integration of equation (20) and the use of boundary conditions (16) gives 


$$
\left.\begin{array}{ll}
p_{00}^{*}=6\left(\frac{H_{1}^{*}-H_{0}^{*}}{H_{1}^{* 3}}\right) x^{*} & 0 \leq x^{*} \leq L_{1}^{*} \\
p_{00}^{*}=p_{c_{1}}^{*}+6 \int_{L_{1}^{*}}^{x^{*}}\left(\frac{H^{*}-H_{0}^{*}}{H^{* 3}}\right) \mathrm{d} x^{*} & L_{1}^{*} \leq x^{*} \leq L_{2}^{*} \\
p_{00}^{*}=6\left(\frac{\left(H_{2}^{*}-H_{0}^{*}\right)\left(x^{*}-1\right)}{H_{2}^{*^{3}}}\right) & L_{2}^{*} \leq x^{*} \leq 1
\end{array}\right\}
$$

where $p_{c_{1}}$ is the dimensionless pressure at $x^{*}=L_{1}^{*}$ and is given by

$$
p_{c_{1}}^{*}=6\left(\frac{H_{1}^{*}-H_{0}^{*}}{H_{1}^{* 3}}\right) L_{1}^{*}
$$

and $H_{0}^{*}=\frac{A}{B}$

where

$$
\begin{aligned}
& A=\frac{L_{1}^{*}}{H_{1}^{* 2}}-\frac{L_{2}^{*}-1}{H_{2}^{* 3}}+\int_{L_{1}^{*}}^{L_{2}^{*}} \frac{1}{H^{* 2}} \mathrm{~d} x^{*} \text { and } \\
& B=\frac{L_{1}^{*}}{H_{1}^{* 3}}-\frac{L_{2}^{*}-1}{H_{2}^{* 3}}+\int_{L_{1}^{*}}^{L_{2}^{*}} \frac{1}{H^{* 3}} \mathrm{~d} x^{*} .
\end{aligned}
$$

Integration of equation (19) and the use of equation (20) gives

$$
\left.\begin{array}{ll}
p_{01}^{*}=\left(-\frac{54}{5}\right)\left(\frac{\left(H_{1}^{*}-H_{0}^{*}\right)^{3}}{H_{1}^{* 7}}\right) x^{*} & 0 \leq x^{*} \leq L_{1}^{*} \\
p_{01}^{*}=p_{c_{2}}^{*}+\left(-\frac{54}{5}\right)_{L_{L}^{*}}^{x^{*}}\left(\frac{\left(H^{*}-H_{0}^{*}\right)^{3}}{H^{* 7}}\right) \mathrm{d} x^{*} & L_{1}^{*} \leq x^{*} \leq L_{2}^{*} \\
p_{01}^{*}=\left(-\frac{54}{5}\right)\left(\frac{\left(H_{2}^{*}-H_{0}^{*}\right)^{3}\left(x^{*}-1\right)}{H_{2}^{* 7}}\right) & L_{2}^{*} \leq x^{*} \leq 1
\end{array}\right\}
$$

where $p_{c_{2}}$ is the dimensionless pressure at $x^{*}=L_{1}^{*}$ and is given by

$$
p_{c_{2}}^{*}=\left(-\frac{54}{5}\right)\left(\frac{\left(H_{1}^{*}-H_{0}^{*}\right)^{3}}{H_{1}^{* 7}}\right) L_{1}^{*} \text {. }
$$

The steady load-carrying capacity of the bearing is calculated by integrating the steady film pressure over the film region

$$
W=B \int_{x=0}^{L} p_{0} \mathrm{~d} x
$$

Expressing in a dimensionless form, one has the steady-state load-carrying capacity in the form

$$
\begin{aligned}
W^{*}=\frac{W h_{m_{0}}^{2}}{\mu U L}= & 3\left(\frac{H_{1}^{*}-H_{0}^{*}}{H_{1}^{* 3}}\right)\left(L_{1}^{*^{2}}-2 L_{1}^{*} L_{2}^{*}\right)\left\{-1+\frac{9 \alpha}{5} \frac{\left(H_{1}^{*}-H_{0}^{*}\right)^{2}}{H_{1}^{*+}}\right\} \\
& +3\left(\frac{H_{2}^{*}-H_{0}^{*}}{H_{2}^{* 3}}\right)\left(L_{2}^{*}-1\right)^{2}\left\{-1+\frac{9 \alpha}{5} \frac{\left(H_{2}^{*}-H_{0}^{*}\right)^{2}}{H_{2}^{*+}}\right\} \\
& +6 \int_{L_{1}}^{L_{2}^{*}}\left[\int_{L_{1}}^{x_{1}^{*}}\left\{\left\{\left(\frac{H^{*}-H_{0}^{*}}{H^{* 3}}\right)\left[-1+\frac{9 \alpha}{5} \frac{\left(H^{*}-H_{0}^{*}\right)^{2}}{H^{*+}}\right]\right\} \mathrm{d} x^{*}\right] \mathrm{d} x^{*}\right.
\end{aligned}
$$

The friction force exerted on the sliding surface is given by

$$
F_{f}=-B \int_{x=0}^{L}\left[\tau_{x y}\right]_{y=0} \mathrm{~d} x
$$

where,

$$
\begin{aligned}
{\left[\tau_{x y}\right]_{y=0} } & =f\left(h, \kappa^{*}, x\right) \\
& =-\frac{1}{h\left(1+\kappa^{*}\left(\frac{h}{2} \frac{\partial p}{\partial x}\right)^{2}\right)}-\frac{h}{2} \frac{\partial p}{\partial x}
\end{aligned}
$$

The dimensionless form of Eq. (25) is as follows

$$
F_{f}^{*}=-\int_{0}^{1} f_{i}\left(H_{i}^{*}, \alpha, x^{*}\right) \mathrm{d} x^{*} \quad \text { for } \quad i=1,2,3
$$

where

$$
\begin{aligned}
& f_{i}\left(H_{i}^{*}, \alpha, x^{*}\right)=-\frac{1}{H_{i}^{*}\left\{1+\alpha\left[\frac{3\left(H_{i}^{*}-H_{0}^{*}\right)}{H_{i}^{* 2}}-\frac{27 \alpha}{5} \frac{\left(H_{i}^{*}-H_{0}^{*}\right)^{3}}{H_{i}^{* 6}}\right]^{2}\right\}} \\
& -\left[\frac{3\left(H_{i}^{*}-H_{0}^{*}\right)}{H_{i}^{* 2}}-\frac{27 \alpha}{5} \frac{\left(H_{i}^{*}-H_{0}^{*}\right)^{3}}{H_{i}^{* 6}}\right]
\end{aligned}
$$

for $i=1,2,3$ and $H_{3}^{*}=H^{*}$.

The coefficient of friction is given by

$$
C^{*}=\frac{F_{f}^{*}}{W^{*}}
$$

2.2. Dynamic stiffness and damping characteristics

With the steady pressure known, the perturbed film pressure can be evaluated from the perturbed Reynolds-type equation (14) with the boundary conditions:

$$
p_{1}^{*}=0 \quad \text { at } \quad x^{*}=0,1
$$

After performing the integration, the perturbed pressure is given by

$$
\begin{array}{ll}
p_{1}^{*}=i \sigma \int_{0}^{L_{1}^{*}} f_{11}^{*} \mathrm{~d} x^{*}+\int_{0}^{L_{1}^{*}} f_{12}^{*} \mathrm{~d} x^{*}+c_{1} \int_{0}^{L_{1}^{*}} f_{13}^{*} \mathrm{~d} x^{*} & 0 \leq x^{*} \leq L_{1}^{*} \\
p_{1}^{*}=i \sigma \int_{L_{1}^{*}}^{x^{*}} f_{21}^{*} \mathrm{~d} x^{*}+\int_{L_{1}^{*}}^{x_{2}^{*}} f_{22}^{*} \mathrm{~d} x^{*}+c_{2} \int_{L_{1}^{*}}^{x_{2}^{*}} f_{23}^{*} \mathrm{~d} x^{*} & L_{1}^{*} \leq x^{*} \leq L_{2}^{*} \\
p_{1}^{*}=i \sigma \int_{L_{2}^{*}}^{1} f_{31}^{*} \mathrm{~d} x^{*}+\int_{L_{2}^{*}}^{1} f_{32}^{*} \mathrm{~d} x^{*}+c_{3} \int_{L_{2}^{*}}^{1} f_{33}^{*} \mathrm{~d} x^{*} & L_{2}^{*} \leq x^{*} \leq 1
\end{array}
$$

where $f_{0}^{*}=\mathrm{d} p_{0}^{*} / \mathrm{d} x^{*}$ and $c_{1}=c_{1 r}+c_{1 i}$. The expressions for $c_{1 r}, c_{1 i}, f_{11}$ etc are as given in the Appendix.

By integrating the perturbed film pressure the perturbed film force is then evaluated

$$
F_{e}=B \int_{x=0}^{L} p_{1} \mathrm{~d} x
$$

The perturbed film force due to the perturbed film pressure is now expressed in terms of linearized stiffness and damping coefficients in accordance with the linearized perturbation theory 


$$
F_{e} \varepsilon e^{i \tau}=-K_{e} h_{m_{0}} \varepsilon e^{i \tau}-D_{e} \frac{\mathrm{d}\left(h_{m_{0}} \varepsilon e^{i \tau}\right)}{\mathrm{d} t}
$$

Expressing in dimensionless quantities results in

$$
F_{e}^{*}=-K_{e}^{*}-i \sigma D_{e}^{*}
$$

Equating the real and the imaginary parts, the dynamic stiffness and damping coefficients are obtained, respectively, as

$$
\begin{aligned}
& K_{e}^{*}=-\operatorname{Re}\left(F_{e}^{*}\right)=\left\{\int_{0}^{L_{0}^{*}} \int_{0}^{*} f_{12}^{*}\left(\mathrm{~d} x^{*}\right)^{2}+\int_{L_{1}}^{L_{L}^{*} x_{1}^{*}} \int_{22}^{*}\left(\mathrm{~d} x^{*}\right)^{2}+\int_{L_{L}}^{1} \int_{L_{2}}^{x_{2}^{*}} f_{32}^{*}\left(\mathrm{~d} x^{*}\right)^{2}\right\} \\
& +\left\{c_{1 r} \int_{0}^{L_{1} x_{0}^{*}} \int_{0}^{*} f_{13}^{*}\left(\mathrm{~d} x^{*}\right)^{2}+c_{2 r} \int_{L_{1}}^{L_{2}^{*} \int_{i}^{*}} \int_{23}^{*}\left(\mathrm{~d} x^{*}\right)^{2}+c_{3 r} \int_{L_{2} L_{2}}^{L_{L_{2}}^{*}} f_{33}^{*}\left(\mathrm{~d} x^{*}\right)^{2}\right\} \\
& D_{e}^{*}=-\frac{\operatorname{Im}\left(F_{e}^{*}\right)}{\sigma}=\left(\frac{1}{\sigma}\right)\left[\left\{\sigma \iint_{0}^{L_{0}^{*} x_{0}^{*}} f_{11}^{*}\left(\mathrm{~d} x^{*}\right)^{2}+\sigma \int_{L_{i}}^{L_{1}} \int_{L_{1}}^{*} f_{21}^{*}\left(\mathrm{~d} x^{*}\right)^{2}+\sigma \int_{L_{2}}^{1} \int_{L_{2}}^{x_{3}^{*}} f_{31}^{*}\left(\mathrm{~d} x^{*}\right)^{2}\right\}\right. \\
& \left.+\left\{c_{i i} \int_{0}^{L_{1} \int_{0}^{*}} \int_{0}^{*} f_{13}^{*}\left(\mathrm{~d} x^{*}\right)^{2}+c_{2 i} \int_{L_{i}}^{L_{2} \int_{i}^{*}} \int_{L_{1}}^{*} f_{23}^{*}\left(\mathrm{~d} x^{*}\right)^{2}+c_{3 i} \int_{L_{2}}^{1} \int_{L_{2}}^{x_{2}^{*}} f_{33}^{*}\left(\mathrm{~d} x^{*}\right)^{2}\right\}\right]
\end{aligned}
$$

\section{Results and discussion}

\subsection{Steady-state characteristics}

Figure 2 shows the variation of non-dimensional steady film pressure $p_{0}{ }^{*}$ with non-dimensional co-ordinate $x^{*}$ for various values of non-linear parameter $\alpha$. It is observed that, at the inlet and outlet of the bearing the pressure $p_{0}{ }^{*}$ is zero. As the value of $x^{*}$ increases the pressure also increases. The critical value $x_{c}{ }^{*}$ of $x^{*}$, is observed at which $p_{0}{ }^{*}$ attains maximum. It is observed that, $p_{0}{ }^{*}$ increases for dilatant and decreases for pseudoplastic lubricants as compared to the Newtonian lubricants. The variation of non-dimensional steady film pressure $p_{0}{ }^{*}$ with non-dimensional co-ordinate $x^{*}$ for different bearing geometries under consideration is plotted in Figure 3. The highest film pressure $p_{0}{ }^{*}$ is observed for inclined stepped bearing found to yield maximum pressure $p_{0}{ }^{*}$ as compared to other bearing geometries.

Figure 4 shows the variation of non-dimensional

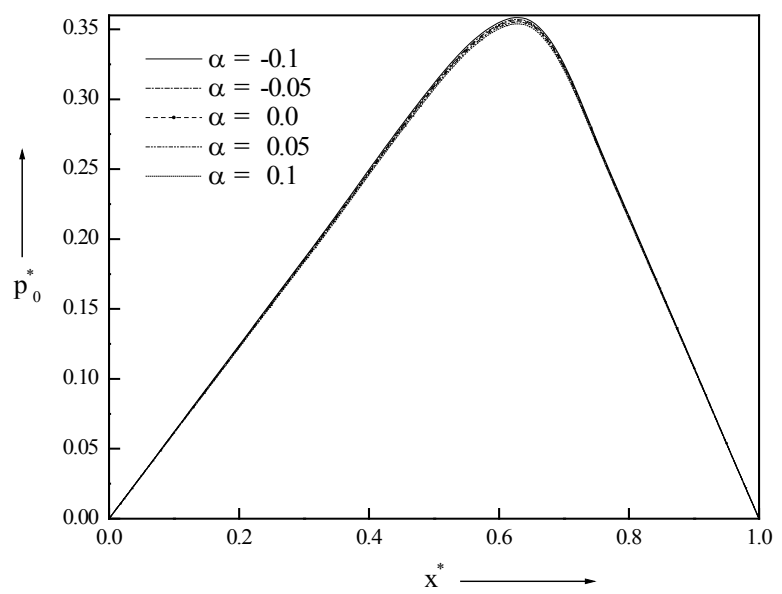

Fig. 2 The variation of non-dimensional film pressure $p_{0}^{*}$ with $x^{*}$ for different values of $\alpha$ with $L_{1}^{*}=0.3$ and $L_{2}^{*}=0.7$ steady load-carrying capacity $W^{*}$ with non-linear factor $\alpha$ for various bearing geometries. Compared to Newtonian lubricants, the load is more delivered for dilatant lubricants and less for pseudoplastic lubricants. Also, it is observed that, the large amount of load is delivered for inclined stepped bearing as compared to the other bearing geometries.

The variation of the co-efficient of friction $C^{*}$ with non-linear parameter $\alpha$ for different types of bearings is depicted in Figure 5. As compared to the other bearings, the lowest coefficient of friction is found for inclined stepped bearing. It is also interesting to note that, in all types of bearings, the coefficient of friction is more for dilatant lubricants and less for pseudoplastic lubricants as compared to the Newtonian case.

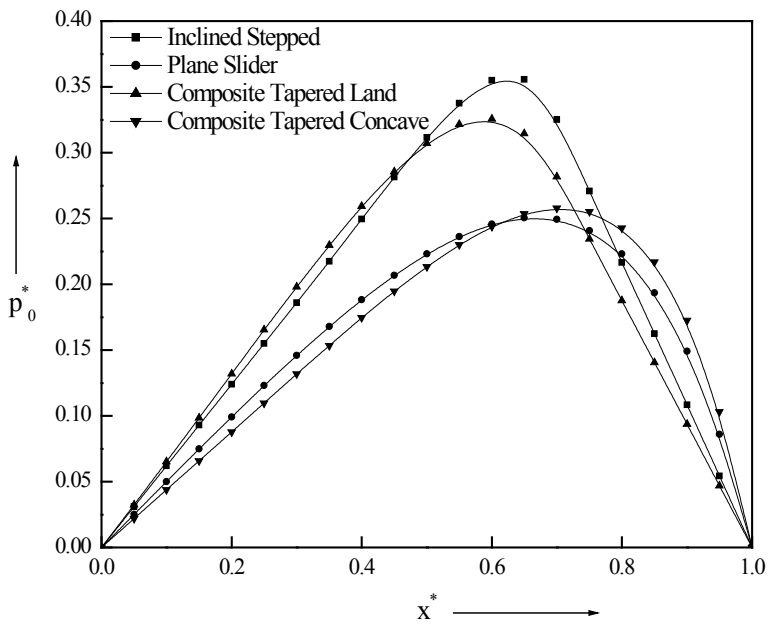

Fig. 3 The variation of non-dimensional film pressure $p_{0}^{*}$ with $x^{*}$ for different bearing geometries with $L_{1}^{*}=0.3$ and $L_{2}^{*}=0.7$

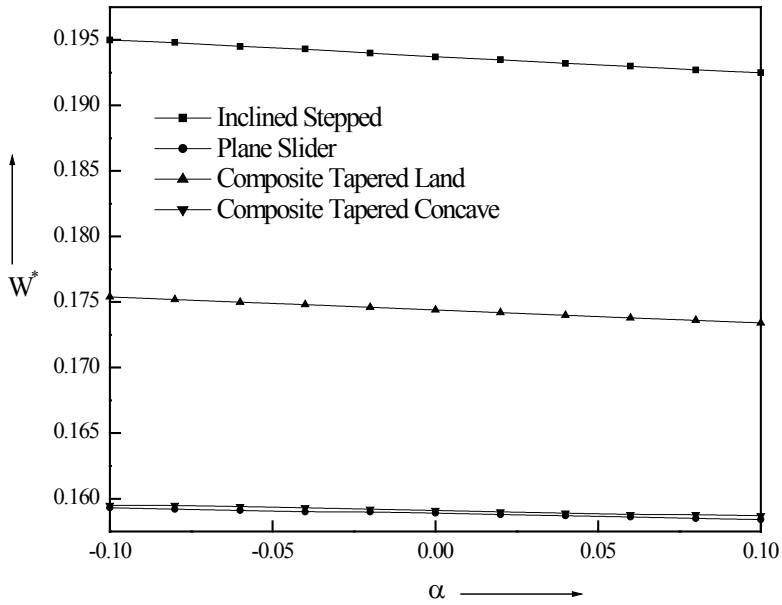

Fig. 4 The variation of non-dimensional load capacity $W^{*}$ with $\alpha$ for different bearing geometries with $L_{1}^{*}=0.3$ and $L_{2}^{*}=0.7$ 


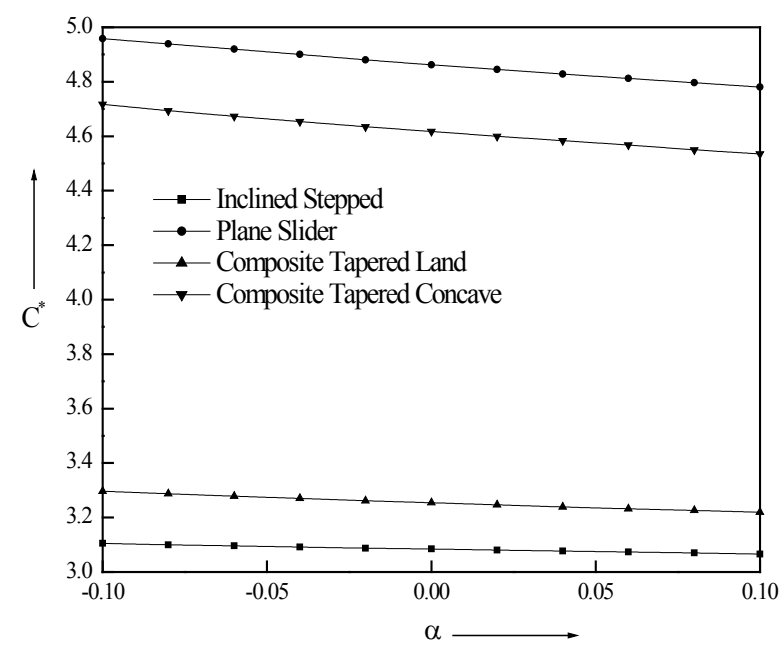

Fig. 5 The variation of coefficient of friction $C^{*}$ with $\alpha$ for different bearing geometries with $L_{1}^{*}=0.3$ and $L_{2}^{*}=0.7$

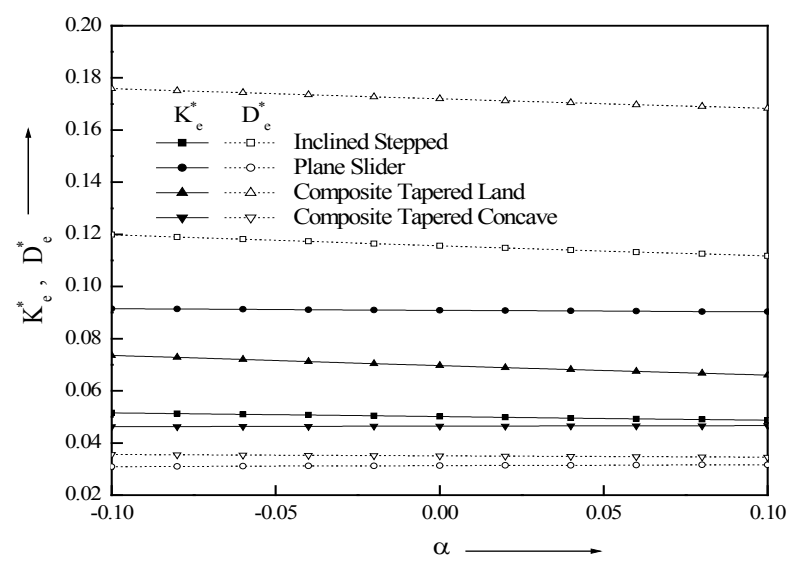

Fig. 6 The variation of non-dimensional dynamic stiffness coefficient $K_{e}^{*}$ and damping coefficient $D_{e}^{*}$ with $\alpha$ for different bearing geometries with $L_{1}^{*}=0.3$ and $L_{2}^{*}=0.7$

\subsection{Dynamic characteristics}

Figure 6 shows the variation of non-dimensional dynamic stiffness coefficient $K_{e}^{*}$ and damping coefficient $D_{e}{ }^{*}$ with non-linear factor $\alpha$ for different types of bearing geometries. It is observed that, among all the bearing geometries under consideration, the $K_{e}^{*}$ is largest $D_{e}{ }^{*}$ is smallest for plane slider bearing. In all types of bearings, it is found that, the dynamic stiffness $K_{e}^{*}$ and damping coefficient $D_{e}{ }^{*}$ decreases for increasing values of $\alpha$.

The variation of non-dimensional dynamic stiffness coefficient $K_{e}^{*}$ with non-dimensional wedge parameter $\delta$ for different bearing geometries is plotted in Figure 7. It

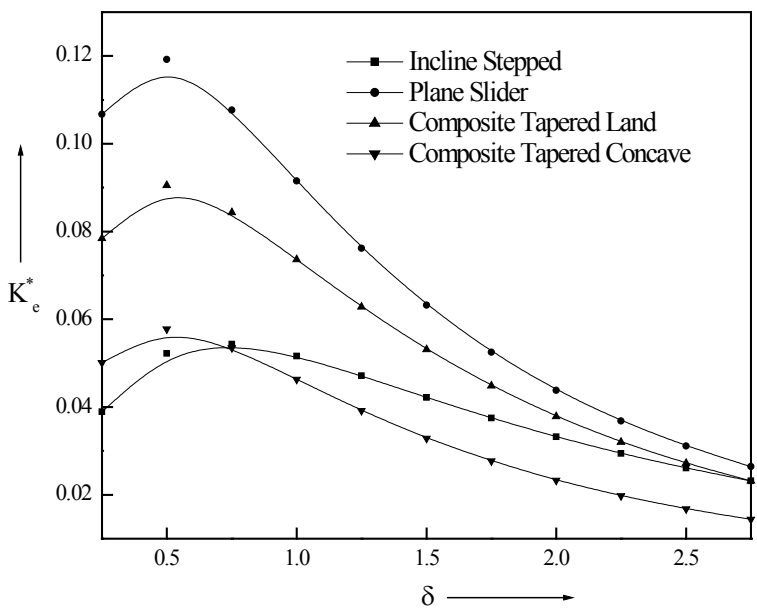

Fig. 7 The variation of non-dimensional dynamic stiffness coefficient $K_{e}^{*}$ with $\delta$ for different bearing geometries with $L_{1}^{*}=0.3$ and $L_{2}^{*}=0.7$

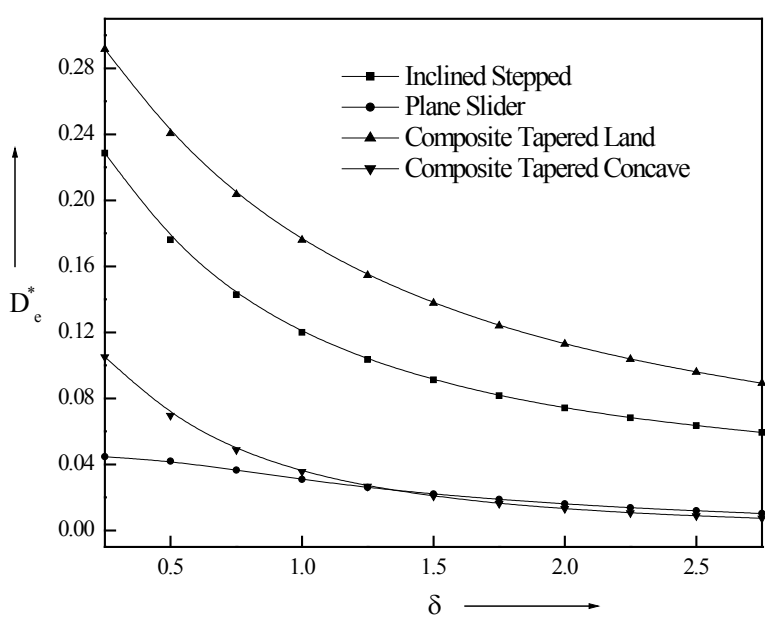

Fig. 8 The variation of non-dimensional damping coefficient $D_{e}^{*}$ with $\delta$ for different bearing geometries with $L_{1}^{*}=0.3$ and $L_{2}^{*}=0.7$

is noted that, the critical value $\delta_{c}$ is observed in all types of the bearings at which the dynamic stiffness coefficient $K_{e}^{*}$ attains maximum. Whereas, on either side of the critical value $\delta_{c}, K_{e}^{*}$ is observed to be decreases gradually. As compared to the other bearings, at the critical value $\delta_{c}$, the maximum dynamic stiffness is obtained for plane slider bearing.

Figure 8 displays the variation of non-dimensional damping coefficient $D_{e}{ }^{*}$ with non-dimensional wedge parameter $\delta$ for different bearing geometries. For small wedge parameter, in all types of bearings, the maximum damping coefficient $D_{e}{ }^{*}$ is observed. Whereas this observation is found more in composite tapered land bearing. 


\section{Conclusions}

On the basis of Rabinowitsch fluid model, this paper predicts the non-Newtonian effects on inclined stepped composite slider bearings. On the basis of the results obtained, the following conclusions can be drawn:

1. The inclined stepped bearing has the largest film pressure than the other geometries. As the lubricant changes from Newtonian to dilatant nature, the film pressure increases, whereas the reverse trend is observed when the lubricant changes from Newtonian to pseudoplastic nature.

2. The highest load-carrying capacity and the lowest coefficient of friction is observed for inclined stepped bearing as compared to the other types of bearings. As compared to the Newtonian lubricants, the load-carrying capacity and coefficient of friction is more for dilatant lubricants and less for pseudoplastic lubricants.

3. As the behavior of the lubricant changes from dilatant to Newtonian and than to pseudoplastic lubricants, the dynamic stiffness and the damping coefficient gradually decreases. The highest dynamic stiffness and the lowest damping coefficient is found for plane slider bearing as compared to the other bearing geometries.

4. The critical value $\delta_{c}$ exists for all types of the bearings at which the dynamic stiffness $K_{e}^{*}$ attains the maximum value.

\section{Nomenclature}

$B \quad$ bearing width

$C^{*} \quad$ coefficient of friction

$d \quad$ a dimension defined in profile function

$D_{e}, D_{e}^{*} \quad$ damping coefficient

$f_{0}^{*} \quad$ pressure gradient

$F_{e}, F_{e}{ }^{*} \quad$ perturbed film force

$F_{f}, F_{f}^{*} \quad$ friction force

$h, h^{*}, H^{*}$ film thickness

$h_{m}, h_{m}{ }^{*} \quad$ minimum film thickness function

$h_{m 0} \quad$ minimum film thickness under steady state

$h_{s}, h_{s}^{*} \quad$ slider profile function

$i \quad \sqrt{-1}$

$K_{e}, K_{e}^{*} \quad$ stiffness coefficient

$L_{1}{ }^{*} \quad$ non-dimensional length of leading parallel portion

$L_{2}{ }^{*} \quad$ sum of the non-dimensional length of leading parallel portion and the inclined protion

$l \quad$ total length of the sliding surface along the direction of motion

$l_{1} \quad$ length of leading parallel portion

$l_{2} \quad$ sum of the length of the leading parallel portion and the inclined portion

$p, p^{*} \quad$ film pressure

$p_{0}, p_{0}{ }^{*} \quad$ steady film pressure

$p_{1}, p_{1}{ }^{*} \quad$ perturbed film pressure

$t, \tau \quad$ time

$u, v \quad$ velocity components of lubricant in the $x$ and $y$ directions respectively

$U \quad$ sliding velocity

$V \quad$ squeeze velocity

$W, W^{*} \quad$ steady load-carrying capacity

$x, y \quad$ cartesian co-ordinates

$x^{*}, y^{*} \quad$ dimensionless co-ordinates

$\alpha \quad$ dimensionless non-linear factor of lubricants

$\delta \quad$ wedge parameter of the slider

$\varepsilon \quad$ small amplitude of oscillation

$\mu \quad$ initial viscosity of a Newtonian fluid

$\sigma \quad$ dimensionless squeeze number

$\kappa \quad$ non-linear factor of lubricants

$\tau_{x y} \quad$ shear stress component

\section{References}

[1] Maiti, G., "Composite and Step Slider Bearings in Micropolar Fluid," Japanese Journal of Applied Physics, 12, 1973, 1058-1064.

[2] Sinha P. and Singh, C., "The Effect of Additives in the Lubricant of a Composite Bearing with an Inclined Stepped Surface,” Wear, 66, 1981, 17-26.

[3] Naduvinamani, N. B. and Apparao, S., "On the Performance of Rough Inclined Stepped Composite Bearings with Micropolar Fluid," Journal of Marine Science and Technology, 18, 2010, 233-242.

[4] Naduvinamani, N. B. and Marali, G. B., "Dyanamic Reynolds Equation for Micropolar Fluid Lubrication of Porous Slider Bearings", Journal of Marine Science and Technology, 16, 2008, 182-190.

[5] Naduvinamani, N. B., Fathima, S. T. and Hiremath, P. S., "Hydrodynamic Lubrication of Rough Slider Bearing with Couple Stress Fluids", Tribology International, 36, 2003, 949-959.

[6] Bhat, M. V. and Deheri, G. M., "Porous Composite Slider Bearing Lubricated with Magnetic Fluid", Japanese Journal of Applied Physics, 30, 1991, 2513-2514.

[7] Shukla, J. B. and Isa, M., "Characteristics of Non-Newtonian Power Law Lubricants in Step Bearings and Hydrostatic Step Seals", Wear, 30, 1974, 51-71.

[8] Wada, S. and Hayashi, H., "Hydrodynamic Lubrication of Journal Bearings by Pseudo-Plastic Lubricants, (Part 2, Experimental Studies)," Bulletin of the JSME, 14, 1971, 279-286.

[9] Bourgin, P. and Gay, B., "Determination of the Load Capacity of a Finite Width Journal Bearing by a Finite-Element Method in the Case of a Non-Newtonian Lubricant", ASME-Journal of Tribology, 106, 1984, 285-290.

[10] Hashimoto, H. and Wada, S., "The Effects of Fluid Inertia Forces in Parallel Circular Squeeze Film Bearings Lubricated with Pseudo-Plastic Fluids," Journal of Tribology, 108, 1986, 282-287. 
[11] Lin, J. R., "Non-Newtonian Effects on the Dynamic Characteristics of One-Dimensional Slider Bearings: Rabinowitsch Fluid Model," Tribology Letters, 10, 2001, 237-243.

[12] Lin, J. R., "Non-Newtonian Squeeze Film Characteristics between Parallel Annular Disks: Rabinowitsch Fluid Model," Tribology International, 52, 2012, 190-194.

[13] Lin, J. R., Hung, C. R., Chu, L. M., Liaw, W. L. and Lee, P. H., "Effects of Non-Newtonain Rabinowitsch Fluids in Wide Parallel Rectangular Squeeze-Film Plates," Industrial Lubrication and Tribology, 65, 2013, (Online Date 27/6/2012) .

[14] He, J. H., "Variational Principle for Non-Newtonian Lubrication: Rabinowitsch Fluid Model," Applied Mathematics and Computation, 157, 2004, 281-286.

[15] Singh, U. P., Gupta, R. S. and Kapur, V. K., "On the Steady Performance of Hydrostatic Thrust Bearing: Rabinowitsch Fluid Model," Tribology Transactions, 54, 2011, 723-729.

[16] Singh, U. P., Gupta, R. S. and Kapur, V. K., "On the Application of Rabinowitsch Fluid Model on an Annular Ring Hydrostatic Thrust Bearing," Tribology International, 58, 2013, 65-70.

[17] Singh, U. P., Gupta, R. S. and Kapur, V. K., "On the Squeeze Film Characteristics between a Long Cylinder and a Flat Plate: Rabinowitsch Model," Proceeding of the Institution of Mechanical Engineers, Part J: Journal of Engineering Tribology, 2012 doi:10.1177/1350650112458742.

\section{Appendix}

The constants in the equation (29) are as given below.

$$
\begin{aligned}
& c_{1 r}=-\frac{\int_{0}^{L_{1}^{*}} f_{12}^{*} \mathrm{~d} x^{*}}{\int_{0}^{L_{1}^{*}} f_{13}^{*} \mathrm{~d} x^{*}} \text { and } c_{1 i}=-\sigma \frac{\int_{0}^{L_{1}^{*}} f_{11}^{*} \mathrm{~d} x^{*}}{\int_{0}^{L_{1}^{*}} f_{13}^{*} \mathrm{~d} x^{*}} \\
& f_{11}^{*}=\frac{240 x^{*}}{H_{1}^{* 3}\left[20+9 \alpha H_{1}^{* 2} f_{0}^{* 2}\right]} ; f_{12}^{*}=-\frac{15 f_{0}^{*}\left[4+\alpha H_{1}^{* 2} f_{0}^{* 2}\right]}{H_{1}^{*}\left[20+9 \alpha H_{1}^{* 2} f_{0}^{* 2}\right]} ; \\
& f_{13}^{*}=\frac{20}{H_{1}^{* 3}\left[20+9 \alpha H_{1}^{* 2} f_{0}^{* 2}\right]} \\
& c_{2}=c_{2 r}+c_{2 i} ; c_{2 r}=-\frac{\int_{L_{1}^{*}}^{L_{2}^{*}} f_{22}^{*} \mathrm{~d} x^{*}}{\int_{L_{1}^{*}}^{L_{2}^{*}} f_{23}^{*} \mathrm{~d} x^{*}} \text { and } c_{2 i}=-\sigma \frac{\int_{L_{1}^{*}}^{L_{2}^{*}} f_{21}^{*} \mathrm{~d} x^{*}}{\int_{L_{1}^{*}}^{*} f_{23}^{*} \mathrm{~d} x^{*}} \\
& f_{21}^{*}=\frac{240 x^{*}}{H^{* 3}\left[20+9 \alpha H^{* 2} f_{0}^{* 2}\right]} ; f_{22}^{*}=-\frac{15 f_{0}^{*}\left[4+\alpha H^{* 2} f_{0}^{* 2}\right]}{H^{*}\left[20+9 \alpha H^{* 2} f_{0}^{* 2}\right]} ; \\
& f_{23}^{*}=\frac{20}{H^{* 3}\left[20+9 \alpha H^{* 2} f_{0}^{* 2}\right]} \\
& c_{3}=c_{3 r}+c_{3 i} ; c_{3 r}=-\frac{\int_{L_{2}^{*}}^{1} f_{32}^{*} \mathrm{~d} x^{*}}{\int_{L_{2}^{*}}^{1} f_{33}^{*} \mathrm{~d} x^{*}} \text { and } c_{3 i}=-\sigma \frac{\int_{L_{2}^{*}}^{1} f_{31}^{*} \mathrm{~d} x^{*}}{\int_{L_{2}^{*}}^{1} f_{33}^{*} \mathrm{~d} x^{*}} \\
& f_{31}^{*}=\frac{240 x^{*}}{H_{2}^{* 3}\left[20+9 \alpha H_{2}^{* 2} f_{0}^{* 2}\right]} ; f_{32}^{*}=-\frac{15 f_{0}^{*}\left[4+\alpha H_{2}^{* 2} f_{0}^{* 2}\right]}{H_{2}^{*}\left[20+9 \alpha H_{2}^{* 2} f_{0}^{* 2}\right]} ; \\
& f_{33}^{*}=\frac{20}{H_{2}^{* 3}\left[20+9 \alpha H_{2}^{* 2} f_{0}^{* 2}\right]}
\end{aligned}
$$

